\title{
Research on Weather Navigation Algorithm Based On Rolling Window Optimization
}

\author{
Qin $\mathrm{Wu}^{\mathrm{a}}$, Yang $\mathrm{Liu}^{\mathrm{b}}$ and Xiaogang $\mathrm{Li}^{\mathrm{c}}$ \\ School of Jilin University, Changchun 130012, China \\ a649239732@qq.com, b578004424@qq.com, c1250737436@qq.com
}

\begin{abstract}
Weather navigation play an important role in real-time sea navigation. The main researches about weather navigation include establish the meteorological model based on the meteorological data, and according to the real-time sensor of marine information combined with weather navigation algorithm plan route dynamically. This paper mainly uses the rolling window optimization algorithm to achieve the weather navigation function. The rolling window optimization algorithm only reference the current environment information and without any prior knowledge of the past and future, so in simulation experiment planning route fall into local oscillation, and lead to weather navigation cannot be completed. In this paper, considered the historical information, a new method which uses the local second optimization sub-target as the local optimization sub-target achieve the idea of global optimal.
\end{abstract}

Keywords: weather navigation; optimization; sub-target; rolling window.

\section{Introduction}

Weather navigation is the study in complex and changeable marine environment on the premise that ensure the safety of navigation [1]. How to select the shortest (or the most provincial fuel) route is the main problem to solve. When the information on global environment has been known, one-time global planning can get a safe path from the start to the end, and optimize some of the performance index in movement process. This has been a very wide range of research.

From the view of practical application, the method of off-line global planning cannot be used because of the lack of knowledge of the real-time weather condition information. Only relying on real-time detection of environmental information and weather information, vessels can get a feasible route after several re- planning (rolling planning). Safety and accessibility of navigation are two main problems.

Based on the predictive control theory of rolling optimization, the problem of vessels weather navigation in the global environment which exist unknown and dynamic dangerous area is studied. An improved rolling window optimization algorithm is proposed, which is based on historical information and future prediction [2,3]. And the algorithm realize combining the optimization with feedback, improve the weather navigation algorithm, and obtain the global dynamic optimal weather route.

\section{Problem description and definition}

Discussing the local route planning problem in the unknown global environment and weather environment which exist dynamic dangerous area, the purpose of the plan is to make the vessels safely navigate from the starting point $P_{\text {start }}$ to the ending point $P_{\text {end }}$. The weather navigation algorithm based on the optimization of the rolling window is driven by a certain time interval and a certain step length. At each step, take the current position of the vessels as the center, with a fixed length for the radius of the rolling window mapping for route planning.

Considering the freedom of the vessels, its limited working area is recorded as $W Z$. According to the actual conditions of the vessels do the following agreements:

(1) Although vessels of the working environment without a priori knowledge, through the GPS sensor can detect the current longitude and latitude coordinates $P_{t}\left(\lambda_{t}, \psi_{t}\right)$ of the vessels.

(2) At any time, vessels can only obtained current position as the center of a circle through the sensor, and radius $r_{m o v}$ of the working area $W Z$ of the environmental information. 
(3) The vessels can be simplified as a particle moving along an arbitrary direction, and the moving step is $\varepsilon_{\text {step }}\left(0<\varepsilon_{\text {step }} \leq r_{\text {mov }}\right)$.

At the same time, the work environment do the following agreements:

(1) In the global environment, there are still a finite number of mutually disjoint static obstacles $O_{S 1}, O_{S 2}, O_{S 3, \ldots,}, O_{S n}$, let $O_{S i} \cap O_{S j}=\emptyset, \mathrm{i} \neq \mathrm{j}$; And there are a finite number of mutually disjoint dynamic dangerous areas $O_{D 1}, O_{D 2}, O_{D 3, \ldots,} O_{D m}, O_{D i} \cap O_{D j}=\emptyset, i \neq j$;

(2) All of the obstacle boundary were expanded, in order to ensure the dangerous area boundary $\partial O_{S i}(i=1,2, \ldots, n), \partial O_{D j}(j=1,2, \ldots, m)$ is also a safe area;

(3) The boundary of the vessels detecting is recorded as $\partial W Z$

Definition 1: Use $d\left(P_{i}, P_{j}\right)$ represent heading distance from point $P_{i}\left(\lambda_{i}, \psi_{i}\right)$ to $\operatorname{point} P_{j}\left(\lambda_{j}, \psi_{j}\right)$. Determined by formula

$$
d\left(P_{i}, P_{j}\right)=\sqrt{\left(\lambda_{i}-\lambda_{j}\right)^{2}+\left(\psi_{i}-\psi_{j}\right)^{2}}
$$

Definition 2: At any $t$ time, $\forall P_{t} \in W Z$, if $P_{t} \notin\left(O_{s i} \cup O_{D j}(t)\right), i=1,2, \ldots n, j=1,2, \ldots, m$, then $P_{t}$ is a feasible point of the vessels at $t$ time. The set of all feasible points at any $t$ time is called feasible zone, recorded $F Z(t)$; Otherwise, at any $t$ time, if $\forall P_{t} \notin W Z$ or $P_{t} \in\left(O_{s i} \cup O_{D j}(t)\right), i=1,2, \ldots, n, j=$ $1,2, \ldots, m$, then $P_{t}$ is an unfeasible point of the vessels at $t$ time.

The set of all unfeasible points at any $t$ time is called unfeasible zone, recorded $D F Z(t)$.

In conclusion, mathematical expressions of feasible zone and unfeasible zone are:

$F Z(t)=W Z \cap\left(\sim U_{i=1}^{n} O_{S i}\right) \cap\left(\sim U_{j=1}^{m} O_{D j}\right)$

$\operatorname{DFZ}(t)=W Z \cap\left(\left(U_{i=1}^{n} O_{S i}\right) \cup\left(U_{j=1}^{m} O_{D j}\right)\right)$

And $\sim U_{j=1}^{m} O_{D j}$ represent the complement of set $U_{j=1}^{m}$.

Definition 3: If $P F Z$ satisfy $P F Z=W Z \cap\left(\sim U_{i=1}^{n} O_{S i}\right) \cap\left(\sim U_{j=1}^{m} O_{D j}\right)$, then call $P F Z$ as permanent feasible zone. If there is any one point $P$, satisfy $P \in P F Z$, then call point $P$ as permanent feasible point.

Definition 4: $\forall P_{S 0}, P_{e 0} \in F Z, t \in[0,1]$, if during time $t$ continuous mapping $F S$ satisfy: $T \rightarrow F S$ letting $F S(0)=P_{S 0}, F S(1)=P_{e 0}$, then Mapping $F S$ is a potentially feasible route from $P_{S 0}$ to $P_{e 0}$ within $W Z$, recorded $F P\left(P_{S 0}, P_{e 0}\right)$. And $P_{S 0}$ is the start point of feasible route. $P_{e 0}$ Is the end point of feasible route? All set of feasible route is called feasible route set, recorded $\Gamma_{F P}\left(P_{S 0}, P_{e 0}\right)$.

Definition 5: At any $t$ time, if there is $\operatorname{Win}\left(P_{t}(t)\right)$ letting itself satisfy $\operatorname{Win}\left(P_{t}(t)\right)=\{P \mid P \in$ $\left.W Z, d\left(p, P_{t}\right) \leq r_{\text {mov }}\right\}$, then call $W i n\left(P_{t}(t)\right)$ as field of view of the vessels at point $P_{t}\left(\lambda_{t}, \psi_{t}\right)$, recorded rolling window for the current moment of the point, and $P_{t}(t) \in F Z(t)$.

\section{Description of the weather navigation algorithm based on the optimization of rolling window}

\subsection{Optimization of rolling window algorithm}

In the optimization of the rolling window algorithm, first, make full use of known environmental information to predict and evaluate the unknown information [4]. Then local optimization is carried out by the form of the rolling window. Last, use feedback information to update the information of the work environment. Repeat the above steps until the vessels is sailing to the end [5]. The steps of the weather navigation algorithm based on the optimization of the rolling window are described as follows.

Step0: Initialize the starting point of the vessels $P_{\text {start }}\left(\lambda_{\text {start }}, \psi_{\text {start }}\right)$, the ending point $P_{\text {end }}\left(\lambda_{\text {end }}, \psi_{\text {end }}\right)$, rolling window working environment $W Z$, radius of sensor detecting $r_{m o v}$ and rolling step $\varepsilon_{\text {step }}$, etc., and $P_{\text {start }}, P_{\text {end }} \in F Z$.

Step1: If the current position $P_{t}\left(\lambda_{t}, \psi_{t}\right)$ has reached the end of $P_{\text {end }}\left(\lambda_{\text {end }}, \psi_{\text {end }}\right)$, end the planning. Else go to Step2.

Step2: According to the data information updated by the sensor, refresh the current environmental information within the rolling window $W Z(t)$. 
Step3: According to the current environment information, combining with the method of heuristic function to generate a local optimization sub-target $P_{b}$ of the current window. And define vector $\overrightarrow{P_{t} P_{b}}$ as a local route planning in the rolling window.

Step4: Vessels go a step along vector $\overrightarrow{P_{t} P_{b}}$ until time $t+1$. Step record $\varepsilon_{\text {step }}\left(0<\varepsilon_{\text {step }} \leq r_{\text {mov }}\right)$.

Step5: Gain data from sensor, then return Step1.

\subsection{Determination of local sub-target}

Due to the final target of global optimization $P_{\text {end }}$ sometimes is not in the rolling window working area, so as it's necessary to produce a local optimization sub-target $P_{b}$ according to the above Step3. $P_{b}$ Can be considered to be the mapping of the target $P_{\text {end }}$ of global optimization in the current rolling window. The production method is as follows.

At any time $t$, the current rolling window optimization for the vessels is $W Z\left(P_{t}(t)\right)$.If $P_{\text {end }} \in$ $W Z\left(P_{t}(t)\right)$, local optimization sub-target satisfy $P_{b}(t)=P_{\text {end }}$. Else use heuristic function $f(P)=$ $g(P)+h(P)$ as performance index function. Select a rolling window boundary point $P$ letting function $f(P)$ get the minimum value as a locally optimal sub-target of this optimization.

$\min f(P)=g(P)+h(P), P \in \partial W Z\left(P_{t}(t)\right)$

$g(P)$ represents the distance from the current position to the window boundary point $P$, and its value can be calculated based on the current position of the vessels and the position of the $P . h(P)$ Represent the true distance from the point $P$ to the end of the vessels. Because the global weather information is often unable to get, $h(P)$ generally represent the course distance from the point $P$ to the end. The selection of this kind of local sub - target is very good, which reflects a balance between the requirement of global optimization and the constraints due to limited local information. The selection of this kind of local sub - target is also a natural selection of global route planning, which is in the environment of some known information.

In order to simplify the calculation and find a feasible route, this paper reduce requirement of the optimization. So the value of $g(P)$ is only determined by whether the $P$ is belong to feasible zone $F Z$

$$
g(P)= \begin{cases}0, & P \in F Z \\ +\infty, & P \notin F Z\end{cases}
$$

In summary, the selection of the local optimal sub-target can be summarized as the following optimization problems:

$\min J=\operatorname{mind}\left(P, P_{\text {end }}\right), P \in \partial W Z\left(P_{t}(t)\right) \cap F Z$

\section{Improve the rolling window optimization algorithm to solve the problem of local oscillation}

\subsection{Problem description}

As shown in Figure 1, vessels choose $\overrightarrow{P_{t} P_{2}}$ as heading from local sub-target at timet. When the window rolls at timet +1 , if still according to formula (6) choose $P_{3}$ as optimal local sub-target from point $P_{3}$ and $P_{4}$, window will roll along $\overrightarrow{P_{t+1} P_{3}}$, then vessels will come back the position at timet. Repeatedly, plan will be into a local oscillation, leading to weather navigation will not be completed.

Except concave polygon, local oscillation will be happened in convex polygons and straight line. There are some differences in rectangle.

As shown in Figure 2, in straight line navigation, after selecting local optimal sub -target according to formula (6), window roll along $\overrightarrow{P_{t} P_{t+1}}$ at time $\mathrm{t}$. When window roll at time $\mathrm{t}+\mathrm{n}$, if still select local optimal sub-target according to formula (6), window will roll along $\overrightarrow{P_{t} P_{t+n-1}}$. And then vessels will go back the position at time $t+n-1$. Repeatedly, plan will be into a straight oscillation, leading to weather navigation will not be completed. 


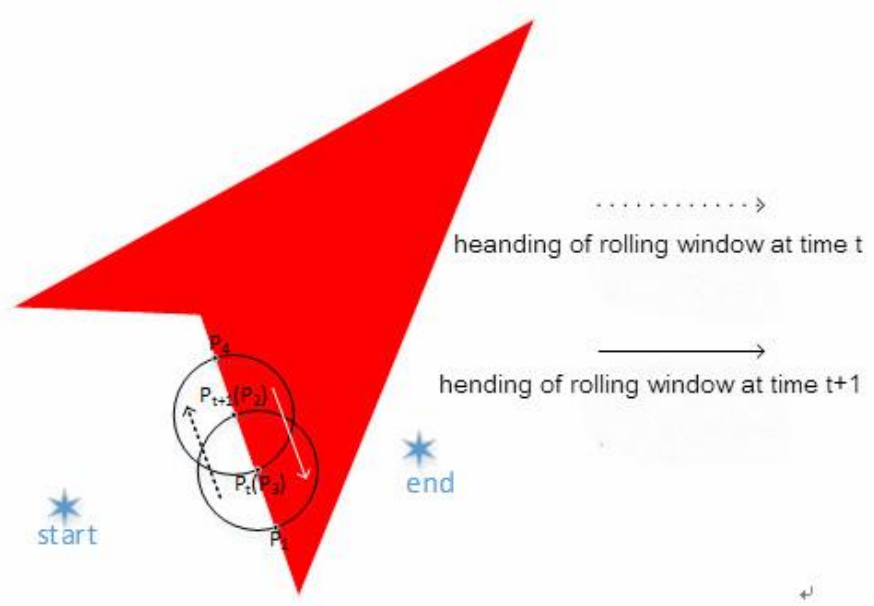

Fig. 1 Schematic diagram of the local oscillation problem in concave polygon

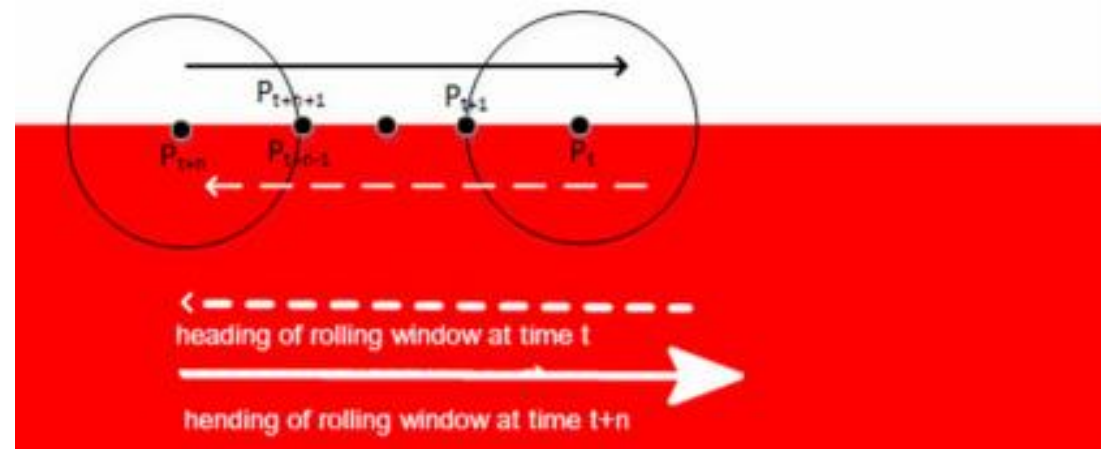

Fig 2 Schematic diagram of the local oscillation problem in straight line navigation

\subsection{Selection of local minimum value and description of algorithm}

In rolling window optimization algorithm, adding some historical information may reach its optimization result. It is impossible that vessels will be in local oscillation if there is only one local optimization sub- target at time t.

If there are two local optimization sub- targets and the local optimization sub- target at time $t$ is the same as the local optimization sub- target at time $t+2$, then we can ensure that the route has been in a local oscillation. It is different for rectangular to be judged whether it is in local oscillation. The method is as follows, assuming $\theta_{t}$ is the angle between $\overrightarrow{P_{t} P_{t+1}}$ and $\overrightarrow{P_{t+1} P_{t+2}}$. If $\theta_{t}=\theta_{t-1}=0^{\circ}$, it means window still rolls at original direction. Else if $\theta_{t}=180^{\circ}, \theta_{t-1}=0^{\circ}$, it means window rolls at opposite direction. And the planning route has been in a local oscillation point.

Step of solving the local oscillation problem, which is based on the improved rolling window optimization algorithm, add a judgement whether vessels is in local oscillation position based on the third section algorithm's Step3. If vessels is in local oscillation position, then select the local second optimization target $\widehat{P_{b}}$ as optimization sub-target of current window. And use vector $\widehat{P_{t} \widehat{P_{b}}}$ as local route planning in the rolling window. If vessels is not in local oscillation position, select the local optimization sub-target $P_{b}$. And use vector $\overrightarrow{P_{t} P_{b}}$ as local route planning in the rolling window.

\section{Simulation results}

In the simulation diagram, the light shadow area polygons represent the unnavigable area of the static coastline. The dark shadow area polygons represent unnavigable areas of bad weather that wind speed is greater than $10 \mathrm{~m} / \mathrm{s}$. Due to the expansion of the boundary of the unnavigable area, the boundary of the unnavigable area is safe area.

Assume the starting point of vessels is $P_{\text {start }}\left(-12.1^{\circ}, 52.5^{\circ}\right)$ and the ending of vessels is $P_{\text {end }}\left(-57.9^{\circ}, 8.4^{\circ}\right)$. Departure time is 0 clock of January 1,2015 . Weather data information is updated every six hours. Area of wind speed greater than $116 \mathrm{~m} / \mathrm{s}$ is dangerous area. Vessels sail at a constant speed in $10 \mathrm{~m} / \mathrm{s}$. Rolling radius is $0.926 \mathrm{~km} / \mathrm{h}$. On the first day, the four session of the 
weather navigation results are shown in Figure 3, the complete weather navigation results are shown in Figure 4.

In this simulation result of weather navigation, window rolls forward about 833 steps, wastes 8 days and 5 hours, plan about $711.358 \mathrm{~km}$. Analysing the picture of simulation, although weather navigation algorithm based on improvement of rolling window optimization maybe not get local optimal sub- target of the current rolling window, ensure global stability and optimal global sub-target in the shortest route, ensure that the vessels can effectively avoid sailing in local oscillation. And help the vessels find a shortest dynamic route.

\section{Summary}

Weather navigation algorithm is a kind of dynamic programming algorithm, which is based on the weather data and real-time information of the ocean environment. In this paper, use the optimization of rolling window algorithm to realize the function of weather navigation. And through improve the algorithm solve one problem. Using the optimization of rolling window algorithm select the nearest point as the current window local optimal sub-target of planning route. Using the local second optimization sub-target as the local optimization sub-target solve local oscillation problem.
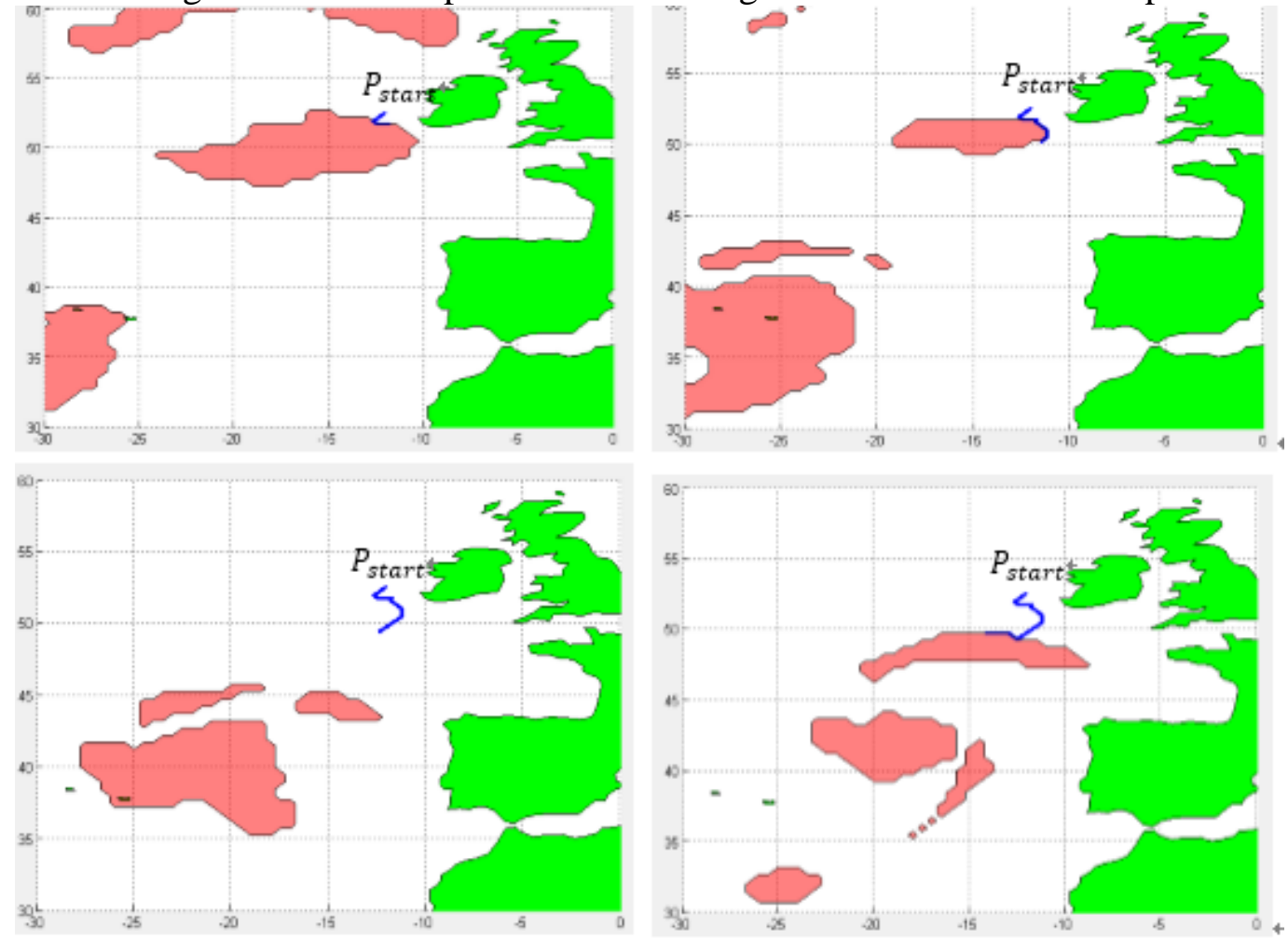

Fig.3 simulation results of weather navigation for 4 time periods in January 1, 2015 


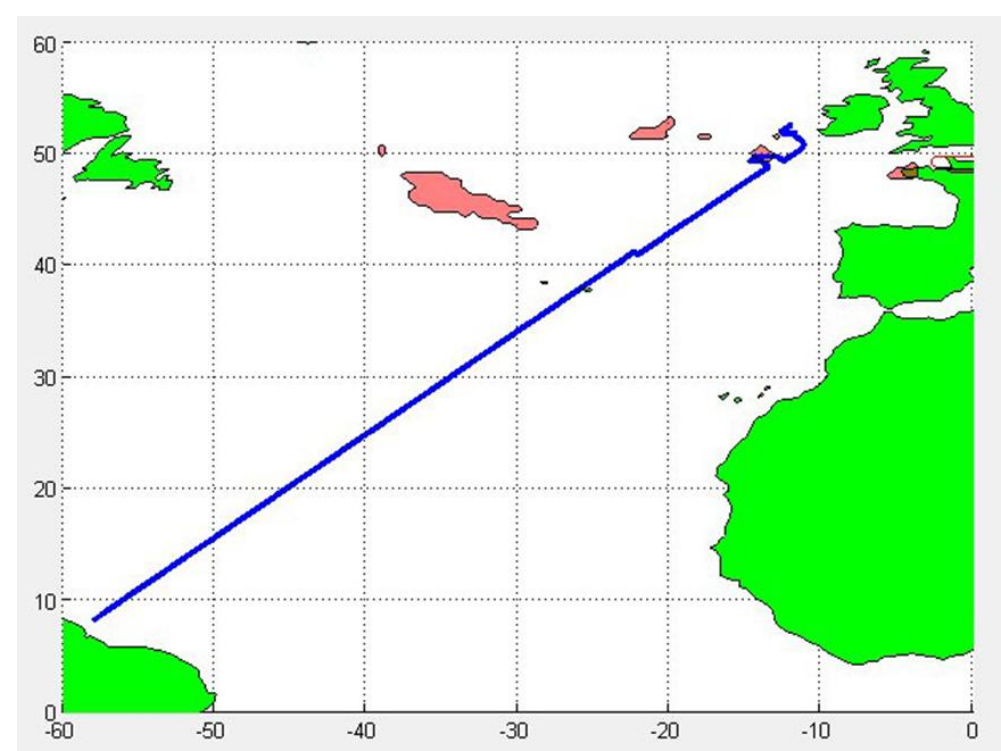

Fig. 4 simulation results of weather navigation algorithm

\section{References}

[1]. .Hagiwara H. Weather routing of (sail-assisted) motor vessels [D]. Delft Technology University, Holland, 1989. p.32-35

[2]. .Yanfeng Chong. Research on path planning method based on rolling optimization principle [D].Jinlin University, China, 2006. p.24-25

[3]. .Zhenyu Wang. Research on path planning algorithm for obstacle avoidance [D]. Huazhong University of Science and Technology, China, 2004. p.12-14

[4]. .Richalet J, Rault A, Testud J L, et al. Model predictive heuristic control: Applications to industrial processes [J]. Automatica, 1978, 14(5): 413-428.

[5]. .Mehra R K, Rouhani R, Rault A, et al. Model algorithmic control: Theoretical results on robustness [C]. Joint Automatic Control Conference. 1979 (16): 387-392. 